\title{
Les avocats tunisiens dans la Tunisie de Ben Ali : économie politique d'une profession juridique
}

\author{
Éric Gobe
}

Centre Jacques Berque (CJB), 35 avenue Tarik Ibn Ziad, 10000 Rabat, Maroc.

<ericgobe@yahoo.fr>

Résumé

L'histoire récente du barreau tunisien est symptomatique des tentatives répétées du régime autoritaire du président Ben Ali à mettre sous tutelle une profession qui, selon le discours de ses représentants, se doit de garantir le respect de l'État de droit et les droits de la défense. Pour ce faire, le pouvoir déchu avait mis en place des dispositifs visant à réduire la capacité de la profession à s'autoréguler. Un premier dispositif consistait à donner aux avocats membres du parti de Ben Ali un accès prioritaire au contentieux des entreprises publiques, tandis qu'un second visait à modeler le territoire professionnel des avocats et des professions voisines en fonction des rapports de force politique et de l'attitude plus ou moins conciliante des instances ordinales vis-à-vis du pouvoir en place.

Avocats - État autoritaire - Samsar - Territoire professionnel - Tunisie.

Summary Lawyers in Ben Ali's Tunisia: The Political Economy of a Legal Profession

The recent history of the Tunisian Bar is symptomatic of the repeated attempts and difficulties encountered by President Ben Ali's authoritarian regime in dominating a profession whose purpose, according to representatives, is to guarantee defendants' rights and respect for the Rule of Law. In order to do this, the power had set up mechanisms to reduce the ability of the profession to self-regulate. A first mechanism was to give those lawyers who were members of Ben Ali's party priority access to litigation cases in state enterprises. A second mechanism was to shape the professional territory of lawyers and related professions according to the political battles of will and more or less conciliatory attitudes of Tunisian Bar towards the political power.

Authoritarian State - Claim of Jurisdiction - Lawyers - Samsar - Tunisia. 
Le 14 janvier 2011, le président Ben Ali quittait la Tunisie après 23 ans d'exercice d'un pouvoir autoritaire et près d'un mois de mouvements protestataires. Par leur participation aux manifestations, les instances dirigeantes du barreau tunisien, comme un grand nombre d'avocats, ont accompagné et apporté leur soutien aux mobilisations populaires. La profession et son bâtonnier en ont tiré un profit symbolique considérable après la chute du régime en place.

Toutefois, il convient de ne pas surévaluer le rôle des avocats dans des mobilisations caractérisées par l'absence de leadership ${ }^{1}$. Les instances ordinales ont d'ailleurs été prudentes au début du mouvement de protestation. Pendant les deux premières semaines du mouvement, ce sont les avocats des villes du centre-ouest et du sud du pays (Sidi Bouzid, Kasserine, Médenine...) puis de Tunis, et non les instances ordinales, qui ont pris l'initiative de participer aux manifestations. Quand le Conseil de l'ordre a lancé un appel à la grève pour le 6 janvier 2011, il l'a fait non pas au nom de la solidarité avec les manifestants, mais pour dénoncer la violence faite aux avocats lors de manifestations à Tunis le 30 décembre 2010.

Ce sont la répression brutale des mouvements protestataires à Kasserine les 7, 8 et 9 janvier 2011 et l'intensification des manifestations qui font prendre conscience aux instances ordinales que la dynamique de l'action collective a pris un tournant radical et concerne toutes les catégories sociales. Le Conseil de l'ordre des avocats décide alors de faire siens les mots d'ordre des Unions régionales de I'UGTT 2 qui, le 11 janvier, ont été autorisées par la direction nationale de la centrale syndicale à organiser des grèves générales sur l'ensemble du territoire tunisien pour " protester contre les tirs à balles réelles sur les citoyens de Sidi Bouzid et Kasserine ». Le 14 janvier au matin, de nombreux avocats revêtus de leurs robes constituent la première ligne des manifestants qui se concentrent devant le ministère de l'Intérieur, avenue Habib Bourguiba. Le départ précipité, le soir même, du président Ben Ali pour l'Arabie Saoudite propulse les avocats sur le devant de la scène de la «Tunisie nouvelle » : ils sont devenus avec leur bâtonnier des « héros de la révolution » qui, héritant d'un fort capital symbolique et politique, fréquentent désormais quotidiennement les plateaux de télévision. Au-delà du déroulement des faits, cette implication de la majeure partie des avocats dans le mouvement protestataire est inséparable de la situation faite à la profession par le régime de Ben Ali.

Dans la configuration autoritaire tunisienne de l'avant-14 janvier, la profession d'avocat était perçue par les gouvernants comme potentiellement dangereuse pour l'ordre politique. II était hors de question pour les pouvoirs publics de permettre à cette profession d'accumuler trop de ressources économiques et politiques. Pour ce faire, le régime de Ben Ali avait mis en place des dispositifs de normalisation. Mais ces dispositifs ont, tout au long des années 2000, montré leurs limites : une large

1. L'absence d'entrepreneurs de mobilisation n'a pas empêché le mouvement de protestation de changer d'échelle. L'usage des nouvelles technologies de l'information a probablement pallié cette absence de leadership en servant de caisse de résonance au mouvement protestataire. Cf. Larbi CHOUIKHA et Éric GOBE, "La force de la désobéissance : retour sur la chute du régime de Ben Ali », in Sarah BEN NEFISSA et Blandine Destremau (dir.), Protestations sociales, révolutions civiles. Transformation du politique dans la Méditerranée arabe, Paris : Armand Colin, 2011 (numéro spécial de la revue Tiers Monde).

2. Union générale tunisienne du travail (UGTT), centrale syndicale tunisienne. 
partie du barreau n'a eu de cesse de rompre avec « la logique du clientélisme politisé, de la discipline et du dressage » qui, selon Béatrice Hibou, sous-tendait les rapports entre l'État autoritaire et les divers groupes sociaux ${ }^{3}$.

Notre objectif, dans cet article, est d'analyser les dispositifs étatiques d'allocation des clients institutionnels qui visaient à contrôler la profession d'avocat dans la Tunisie de Ben Ali et de comprendre la signification politique de certaines pratiques et revendications économiques de la profession. L'approche est donc celle d'une économie politique du barreau tunisien. Elle s'appuie sur les données d'une enquête quantitative et qualitative menée à une large échelle auprès des avocats tunisiens en exercice.

\section{L'enquête}

Elle a été effectuée auprès d'un échantillon de 626 avocats (environ $10 \%$ de la population des avocats, stagiaires compris en 2008) représentatif des grandes agglomérations tunisiennes qui concentrent à elles seules plus de $90 \%$ des avocats ${ }^{4}$. Nous avons mené l'enquête auprès des avocats du Grand Tunis, de Sousse, de Monastir, de Sfax, autrement dit les principales agglomérations de Tunisie. Nous avons également récolté une vingtaine de questionnaires auprès d'avocats de Siliana, Bizerte, Kairouan et Tataouine. Sur les 626 questionnaires, près de 500 ont été collectés lors de séminaires, conférences et assemblées générales organisés respectivement par le Conseil de l'ordre des avocats, les sections de Tunis, de Sfax et de Sousse. Ensuite, une soixantaine de questionnaires ont été distribués et récupérés à Sousse par des étudiants de l'Institut de comptabilité et d'administration des entreprises. J'ai moi-même fait directement remplir une soixantaine de questionnaires lors des entretiens qualitatifs effectués lors de mes séjours en Tunisie en 2008 et au premier semestre de l'année 2009. Le guide d'entretien se structure, outre les questions d'état civil et de parcours scolaire, autour du domaine d'activité de l'avocat, de son travail, de sa clientèle et de l'organisation de son cabinet. Nous avons également posé des questions d'opinion se rapportant au fonctionnement de la justice tunisienne, à la possible concurrence d'autres professions et aux mesures susceptibles d'améliorer le fonctionnement du barreau. Par ailleurs, de la fin 2005 à 2009, chevauchant l'enquête quantitative, j'ai conduit des entretiens qualitatifs (85) dans lesquels je demandais aux avocats de me parler de leur carrière, de leur pratique professionnelle et de leur perception de la situation de la profession. L'objectif des deux types d'enquête était de recueillir des données permettant de comprendre les logiques politique, économique et sociale régissant le fonctionnement de la profession. L'échantillon constitué dans le cadre de l'enquête qualitative n'est pas représentatif, mais nous avons tenté de couvrir le spectre des situations socio-économiques auxquelles sont confrontés les avocats tunisiens (la localisation géographique des divers cabinets donne un premier indicateur). J'ai interviewé des avocats généralistes comme des avocats spécialisés dans divers domaines du droit. Mon échantillon qualitatif comprend 19 avocats spécialistes (14 en droit des affaires, 2 pénalistes et 3 avocats dont l'activité dominante se rapporte respectivement au droit immobilier, aux droits du travail et de la famille), 61 avocats généralistes, 3 stagiaires rémunérés et 2 stagiaires non rémunérés. $80 \%$ des avocats rencontrés sont installés à Tunis et dans ses banlieues.

3. Béatrice HIBOU, La force de l'obéissance. Économie politique de la répression en Tunisie, Paris : La Découverte, 2006, p. 142.

4. Nous avons eu recours à la méthode par quotas. Nous avons utilisé comme premier critère de stratification les tribunaux près lesquels les avocats sont inscrits (Cour de cassation, cour d'appel et stagiaire). Ensuite, nous avons tenu compte de la répartition géographique des avocats selon la section où ils sont inscrits (Tunis, Sousse et Sfax). 
Dans la Tunisie de Ben Ali, le premier dispositif mis en œuvre ressortissait à l'allocation clientéliste des ressources contrôlées par l'État: par l'intermédiaire du parti du président de la République, l'oligarchie au pouvoir distribuait aux avocats lui ayant fait allégeance la clientèle constituée par les institutions publiques. En revanche, elle punissait les avocats qui affichaient des velléités d'opposition en les empêchant d'avoir accès à une clientèle institutionnelle, publique comme privée.

Un second dispositif ne relevait pas d'une intervention directe de l'État autoritaire. Au contraire, il se caractérisait par une forme de laisser-faire qui servait à la fois de soupape de sûreté et d'instrument de contrôle d'une partie de la profession. II permettait aux avocats généralistes les moins pourvus en capital social d'avoir recours à des intermédiaires illégaux pour constituer leur clientèle. Or, ces courtiers chargés de rabattre les clients vers les avocats étaient souvent des agents de l'appareil répressif (policiers, gendarmes, greffiers...). En se mettant dans une situation de dépendance, les avocats qui recouraient au service de ces agents s'exposaient ainsi à une éventuelle punition de la part des pouvoirs publics.

Un troisième dispositif mis en place par le régime de Ben Ali était d'ordre législatif. II consistait à modeler le territoire professionnel des avocats et des professions voisines en fonction des rapports de force politique et de l'attitude plus ou moins conciliante des instances ordinales vis-à-vis du pouvoir politique.

Avant d'aborder dans le détail ces divers dispositifs, il convient de fournir quelques données de cadrage sur les principales modalités de la formation des avocats et de l'exercice de la profession dans la Tunisie de Ben Ali.

\section{Formation des avocats et formes d'exercice de la profession : éléments d'analyse}

Les avocats en Tunisie constituent une profession qui fonctionne selon un modèle libéral et individualiste. La salarisation est quasiment inexistante et les avocats pratiquent massivement à titre individuel (près de $90 \%$ ). Le nombre de sociétés d'avocats est très faible (83 en 2007) et regroupe moins de $10 \%$ des avocats inscrits près les cours d'appel et de cassation. Par ailleurs, le nombre de salariés ayant le statut de collaborateur et travaillant au sein de sociétés d'avocats est très faible. Ces petites structures avec peu d'associés ne comptent pas plus de deux ou trois avocats, parfois membres de la même famille. Ce sont des entreprises fermées 5 avec peu d'employés : $78 \%$ des sociétés ont de 1 à 4 employés. De manière générale, les cabinets tunisiens ont peu de personnel : près de $80 \%$ disposent soit d'une secrétaire et d'un clerc chargé de faire les courses au tribunal, soit d'une personne qui remplit les deux fonctions.

L'immense majorité des avocats tunisiens sont des généralistes qui représentent à la fois des clients individuels et, dans une moindre mesure, des entreprises et qui pratiquent la plupart des domaines du droit. Seul un cinquième des avocats se considèrent comme des spécialistes. L'opposition entre avocats généralistes et avocats

5. Leur capital n'est pas destiné à s'ouvrir aux stagiaires. 
spécialistes et surtout entre les praticiens des affaires et les autres est structurante de la hiérarchisation du barreau tunisien en termes de revenus. Elle est la conséquence du type de clientèle et du domaine de droit pratiqué. La spécialité dominante est le droit des affaires (plus de la moitié des spécialistes ${ }^{6}$ ), c'est-à-dire le domaine du droit qui permet aux avocats de se situer au sommet de la hiérarchie des chiffres d'affaires 7 . Ces derniers constituent le segment dominant du barreau : on verra plus loin qu'ils produisent un discours libéral et élitiste sur la profession.

La profession a connu au cours des 15 dernières années une forte massification en raison de la croissance continue du nombre d'étudiants en droit et de la multiplication des troisièmes cycles juridiques. Le barreau tunisien est devenu au cours des années 2000 l'un des principaux débouchés des étudiants en droit, d'autant que le recrutement des diplômés dans la fonction publique s'est fortement tari.

\section{Graphique 1}

\section{Évolution du nombre d'avocats en Tunisie depuis 1971}

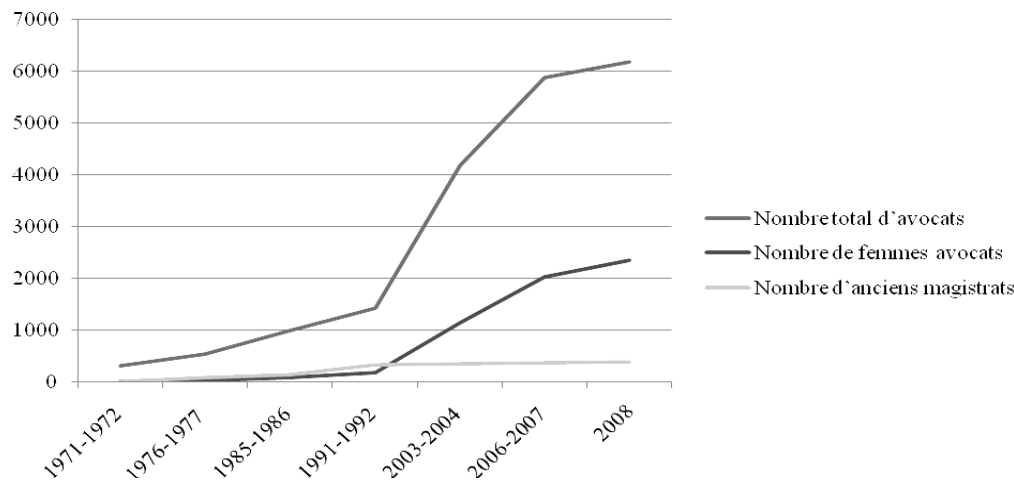

Source : Annuaire des avocats

6. Ensuite, on trouve les spécialistes en droit immobilier (14\%), en droit pénal (12\%), en droit civil $(9 \%)$, en matière de statut personnel ( $5 \%$ ) et en droit du travail ( $5 \%)$.

7. Dans notre enquête qualitative, nous avons demandé aux avocats rencontrés de nous communiquer leur chiffre d'affaires. Ce sont, de loin, ceux spécialisés en droit des affaires qui enregistrent les chiffres d'affaires les plus élevés. Au sommet de la pyramide, on trouve quatre des sociétés tunisiennes d'avocats les plus importantes, dont le chiffre d'affaires varie entre 1 et 5 millions de dinars tunisiens (DT, 10 dinars équivalant à peu près à 6 euros). Viennent ensuite huit avocats d'affaires exerçant à titre individuel et un avocat exerçant en société, avec des chiffres d'affaires oscillant entre 100000 et 500000 DT. On notera qu'un spécialiste en droit immobilier et un en droit du travail s'intercalent dans le classement, avec respectivement des chiffres d'affaires de 150000 et 200000 DT. Deux avocats membres du parti au pouvoir, le RCD, ont des chiffres d'affaires qui se situent entre 100000 et 120000 DT (spécialité en droit des affaires avec une dominante dans les assurances). Au bas de l'échelle des chiffres d'affaires des spécialistes, on trouve deux pénalistes (100 000 et 60000 DT) et une spécialiste en droit de la famille (50 000 DT). Les 43 généralistes qui nous ont fourni l'information ont généralement des chiffres d'affaires moins élevés puisqu'ils s'échelonnent de 10000 à 70000 DT (le salaire minimum tunisien était en 2010 d'environ 300 DT, le salaire moyen d'un enseignant du secondaire en milieu de carrière était de 800 DT). 
Les avocats, comme d'autres professions en Tunisie, sont par conséquent touchés par la massification du système de l'enseignement supérieur : entre 1980 et 1992, le nombre d'avocats s'est accru de $96 \%$, passant de 707 à 1429 ; puis entre 1992 et 2004, il a augmenté de $200 \%$; et entre 2004 et 2008, de plus de $30 \%$ (graphique 1). La population totale tunisienne n'a évidemment pas crû au même rythme que celle des avocats (environ 6 millions d'habitants en 1985 contre près de 10 millions en 2007). Entre 2008 et le début de 2011, le barreau a vu l'inscription de 1500 avocats supplémentaires.

Cet accroissement annuel du nombre d'arrivants s'explique par le caractère peu sélectif de la filière. En effet, il existait jusqu'en 2008 deux voies pour accéder à la profession : la voie classique du CAPA, c'est-à-dire le certificat d'aptitude à la profession d'avocat, examen passé directement après la maîtrise ; et celle du DEA, rendu plus aisé par la multiplication des troisièmes cycles de droit en Tunisie.

On a donc affaire à une population jeune. Elle est, de plus, en voie de féminisation. La profession comptait 3 femmes en 1971-1972, contre 35 en 1979-1980 (5\% du total des avocats), 1136 en 2003-2004 (27\%), et 2338 en 2008 (37,5\%). Par ailleurs, les stagiaires 8 constituaient, en 2010 , près de $40 \%$ de la population des avocats, ce qui est énorme et contribue à alimenter le discours sur la pression que les jeunes avocats exercent sur le marché des services juridiques. À partir du début des années 1990, les instances dirigeantes de l'Ordre ont, dans une logique malthusienne, revendiqué l'unification des conditions d'accès à la profession pour contrôler les flux d'entrants. Pour ce faire, ils ont exigé la mise en place d'un Institut supérieur du barreau. Dans l'esprit des représentants de la profession, cet établissement de formation contrôlé par le Conseil de l'ordre devait permettre de durcir les conditions de recrutement. Le projet élaboré en 2004 par les instances dirigeantes de la profession prévoyait des dispositions consacrant l'autonomie de l'Institut à l'égard des pouvoirs publics. Mais la loi votée par le Parlement le 9 mai 2006 s'est éloignée du texte élaboré par le Conseil de l'ordre en faisant de l'Institut un établissement public placé sous la double tutelle du ministère de la Justice et des droits de l'homme et du ministère de l'Enseignement supérieur. Ce faisant, les gouvernants ont montré qu'ils n'étaient pas prêts à déléguer la gestion du flux d'avocats et leur formation aux structures dirigeantes de l'Ordre ${ }^{9}$. Ils n'avaient pas l'intention de renoncer à la possibilité de déterminer les flux d'entrée dans la profession et d'agir ainsi sur « la production des producteurs $\gg 10$.

Par ailleurs, $42 \%$ des avocats ont exercé une autre profession avant de s'inscrire au tableau de l'Ordre ${ }^{11}$. La profession continue d'exercer un pouvoir d'attraction en

8. Le titulaire d'un DEA (mastère) ou du CAPA pouvait jusqu'en 2009 demander à être inscrit au tableau des avocats en exercice en produisant un certificat d'admission de stage dans le cabinet d'un avocat inscrit près la Cour de cassation ou inscrit près la cour d'appel depuis au moins trois ans. La durée du stage est de deux années minimum et peut être prolongée.

9. L'Institut a commencé à fonctionner pendant l'année judiciaire 2008-2009.

10. Richard L. ABEL, "Comparative Sociology of Legal Profession », in Richard L. ABEL. et Philip S. C. LEWIS (eds.), Lawyers in Society, vol. 3 : Comparative Theories, Berkeley: University of California Press, 1989, p. 80-153.

11. Source : enquête Éric Gobe, 2008-2009. 
dépit de ses difficultés économiques. En effet, les avocats stagiaires qui ont occupé un emploi avant de devenir avocat sont entrés, pour la majorité d'entre eux, dans la profession en raison du manque de perspectives professionnelles. Contrairement à leurs aînés, ils commencent leur vie professionnelle par une longue période de chômage ou en occupant des emplois instables dans les secteurs privé et public 12 . Tenter sa chance au barreau apparaît alors aux diplômés en droit comme une solution alternative.

De leur côté, les plus anciens avocats, ceux inscrits près la Cour de cassation, ont pour $60 \%$ d'entre eux occupé un emploi dans le secteur public avant de devenir avocats : l'intégration au barreau leur a souvent permis d'accéder tout à la fois à plus d'autonomie et à des revenus financiers supérieurs à leur salaire antérieur ${ }^{13}$. Ils ont pu également convertir en clientèle le capital social accumulé pendant leur exercice professionnel dans le secteur public. Mais il convient de noter que certains se sont vus écartés de l'accès au contentieux des institutions et entreprises publiques à la suite de prises de position plus ou moins critiques à l'égard du régime de Ben Ali. Un membre d'un parti d'opposition reconnu, le Tajdid (l'ancien Parti communiste tunisien), avocat depuis dix ans, évoque l'impossibilité pour les opposants de représenter les institutions publiques sous Ben Ali :

J'ai participé à des élections dans les années 1990. J'ai été un peu sanctionné, mais je n'ai pas été licencié, on m'a enlevé quelques avantages. Finalement, le marché des avocats est très contrôlé. À titre d'exemple, j'ai été directeur juridique à la Sécurité sociale. Ca fait 10 ans que je suis parti et je n'ai pas eu une seule affaire de cette boîte. Parce que ces décisions-là sont prises à un certain niveau 14 .

Pour les avocats engagés dans l'opposition, le barreau a pu constituer un refuge pour échapper aux sanctions professionnelles ou disciplinaires prises par l'employeur public. En effet, plusieurs militants connus pour leur engagement contre le régime de Bourguiba et ayant perdu leur emploi dans le secteur public ou dans les médias en raison de leurs activités syndicale et politique sont devenus avocats 15 .

Ces derniers exemples montrent combien l'avocature était considérée par les gouvernants comme un secteur professionnel sensible qu'il convenait, dans la me-

12. Sur l'ampleur du chômage des diplômés et les difficultés d'insertion sur le marché de l'emploi, il convient de consulter la dernière enquête de la Banque mondiale sur la dynamique de l'emploi des jeunes en Tunisie. Elle montre que le chômage affecte de plus en plus les diplômés du supérieur (leur nombre a presque doublé en 10 ans ; ils étaient 336000 au cours de l'année 2006-2007 contre 121800 en 1996-1997). C'est dans les filières du tertiaire (gestion, finances, droit) que le taux de chômage est le plus élevé, atteignant 68 \% pour les titulaires d'une maîtrise en droit, 18 mois après l'obtention de leur diplôme. Cf. le document conjoint : BANQUE MONDIALE et MINISTERE TUNISIEN DE L'EMPLOI ET DE L'INSERTION PROFESSIONNELLE DES JEUNES, Dynamique de l'emploi et adéquation de la formation parmi les diplômés universitaires, vol. 1 : Rapport sur l'insertion des diplômés de l'année 2004, Tunis, 2008.

13. Source : enquête Éric Gobe, 2008-2009.

14. Entretien, mai 2008

15. Tel est le cas de trois des avocats que nous avons rencontrés : l'un est un ancien journaliste, membre de la Ligue tunisienne des droits de l'homme (LTDH) ; le second était cadre dans une compagnie d'assurances, militant d'extrême gauche et membre de l'UGTT ; le troisième était enseignant et militant au sein du syndicat de l'enseignement secondaire de l'UGTT. On notera que ces trois personnalités sont liées par des relations amicales, professionnelles et politiques très étroites. Les deux premiers sont associés dans le même cabinet d'avocats et le dernier a été leur stagiaire. Tous trois sont membres de longue date de la LTDH (entretiens réalisés en novembre 2005 et en novembre 2006). 
sure du possible, de contrôler. Pour ce faire, les autorités récompensaient et sanctionnaient les uns et les autres dans une logique clientéliste.

\section{De la clientélisation de la profession}

L'État autoritaire régulait pour partie directement la distribution de la clientèle aux avocats en fonction de critères politiques. En effet, par le biais du parti-État, le Rassemblement constitutionnel démocratique ( $R C D$, dont le président n'était autre que le chef de l'État), les gouvernants distribuaient en priorité aux professionnels qui les servaient la représentation des institutions de l'État et des entreprises publiques.

Plusieurs avocats interrogés, qu'ils soient membres de l'opposition ou se décrivant comme "professionnels ", parlaient, à propos de la distribution des représentations des sociétés publiques et du contentieux de l'État par le RCD, de " grande samsara " (al-samsara al-koubra) ${ }^{16}$. Le bureau politique du parti servait d'intermédiaire, de courtier (samsar) entre les avocats, les patrons du secteur public et les gouvernants (le président de la République et son entourage) en fixant les listes des avocats autorisés à représenter les institutions étatiques. Les hiérarques du parti, et plus particulièrement le président de la République, accordaient aux " avocats méritants " gratifications matérielles et symboliques. Pour être inscrit sur ces listes, il fallait au minimum être un membre actif de la cellule professionnelle des avocats, la khaliyya. Cette dernière, qui dépendait directement du bureau politique du RCD et du président Ben Ali, n'était pas soumise, contrairement aux autres cellules du parti, à la règle de l'élection 17 .

Par l'intermédiaire de ce clientélisme institutionnalisé, symptôme de l'étatisation de la société ${ }^{18}$, le régime s'assurait du soutien politique d'une partie de la profession en contrepartie de gratifications matérielles.

Les trois quarts des avocats rencontrés dénonçaient l'existence d'un monopole des avocats membres du RCD sur le contentieux du secteur public. Mais quelquesuns des avocats sans affiliation partisane $(3,5 \%)$ avaient accès au contentieux de l'État et des sociétés publiques (tableau 1).

Dans notre échantillon, à l'exception d'un membre du Mouvement des démocrates socialistes, l'un des partis de l'opposition faire-valoir satellisé par le pouvoir présidentiel 19, les avocats membres de l'opposition légale n'avaient pas affaire à des sociétés publiques ou à des administrations.

Pour autant, appartenir au RCD ne signifiait pas nécessairement avoir des institutions publiques dans sa clientèle puisque plus de $40 \%$ des avocats membres du parti présidentiel avaient uniquement des individus ou des sociétés privées dans leur

16. Abderraouf AYADI, "Les informations dignes de foi concernant les tribulations des avocats », document (en arabe) non publié. L'auteur (avocat militant d'extrême gauche) distribuait son texte dans l'hôtel où se déroulait l'élection du bâtonnier de l'Ordre des avocats le 20 juin 2010.

17. Chawki TABIB, Avocats et politique en Tunisie. Étude empirique, mémoire de mastère en sciences politiques, Faculté de droit et de sciences politiques de Tunis, 2006, p. 50-51.

18. Jean-Yves Dormagen, Logiques du fascisme. L'État totalitaire en Italie, Paris : Fayard, 2008, p. 210-252.

19. Michel CAmAu et Vincent GeISSER, Le syndrome autoritaire. Politique en Tunisie de Bourguiba à Ben Ali, Paris : Presses de Sciences Po, 2003. 
portefeuille 20. Certains jeunes avocats membres du RCD espéraient accéder aux institutions publiques, mais se plaignaient qu'au sein du parti certains disposent d'un monopole sur la représentation de l'administration et des entreprises étatiques 21 .

Tableau 1

Part du secteur public dans le chiffre d'affaires des avocats inscrits près la Cour d'appel et de cassation selon l'appartenance ou non au RCD (en \%, $n=369$ )

\begin{tabular}{|l|c|c|c|}
\hline $\begin{array}{l}\text { Part du secteur } \\
\text { public dans le chiffre } \\
\text { d'affaires }\end{array}$ & $\begin{array}{l}\text { Sans affiliation partisane } \\
\text { ou membre d'un parti de } \\
\text { ''opposition }\end{array}$ & Membre du RCD & Total \\
\hline 61 à $80 \%$ & 0 & 1,5 & 0,5 \\
\hline 41 à $60 \%$ & 0 & 9 & 1,5 \\
\hline 21 à $40 \%$ & 0,5 & $\mathbf{2 3}$ & 4 \\
\hline 5 à $20 \%$ & 3 & $\mathbf{2 5}$ & 7 \\
\hline $0 \%$ & 96,5 & $\mathbf{4 1 , 5}$ & 87 \\
\hline Total & 100 & 100 & 100 \\
\hline
\end{tabular}

Source : enquête Éric Gobe 2008-2009.

Le bureau politique du RCD procédait à la sélection des avocats membres de la cellule qui pouvaient bénéficier de la manne publique. Pour prétendre à ce privilège, l'avocat membre du RCD devait avoir des états de service suffisants. Lors d'une première étape, il convenait d'être un militant actif de la khaliyya, autrement dit de participer aux activités associatives du parti pour faire la preuve de son allégeance et espérer ainsi voir à l'avenir une institution publique recourir à ses services 22 . Les militants du RCD adhéraient d'ailleurs aux associations non professionnelles dans des proportions plus grandes que les avocats membres d'un mouvement d'opposition ou sans appartenance partisane (tableau 2). Celles-ci étaient pour la plupart des organisations satellites du parti présidentiel 23.

Ainsi se jouait une compétition féroce entre les jeunes membres du RCD qui s'efforçaient d'obtenir une clientèle privilégiée. Ceux qui pouvaient avoir recours au parrainage d'un hiérarque du parti ou mieux encore du président de la République ou de son entourage familial voyaient leur clientèle publique grossir ${ }^{24}$. Au patronage de l'appareil partisan se superposait celui plus personnalisé des « hommes forts » du régime de Ben Ali.

20. Le pourcentage est encore plus élevé si l'on inclut les stagiaires (près de $60 \%$ ).

21. Entretien avec un jeune avocat membre du RCD, avocat près la cour d'appel depuis moins d'un an, octobre 2009

22. Id.

23. Ce que les Tunisiens appelaient sur le mode humoristique les «OVG " (organisations vraiment gouvernementales). On citera à titre d'exemples, l'Association de développement du micro-crédit, l'Association des mères tunisiennes, l'Association de soutien aux handicapés, l'Association de développement de la ville de Kairouan, l'association Avocats sans frontières, etc.

24. Abderraouf AYADI, «Les informations dignes de foi concernant les tribulations des avocats », op. cit. 
Tableau 2

Taux d'adhésion à une association non professionnelle selon son appartenance au RCD $(n=613)$

\begin{tabular}{|l|c|c|c|}
\hline & $\begin{array}{c}\text { Sans affiliation partisane } \\
\text { ou membre d'un parti } \\
\text { de l'opposition }\end{array}$ & Membre du RCD & Total \\
\hline $\begin{array}{l}\text { Non-affiliation à une } \\
\text { association }\end{array}$ & 80 & 59 & 77 \\
\hline $\begin{array}{l}\text { Affiliation à une } \\
\text { association }\end{array}$ & 20 & 41 & 23 \\
\hline Total & 100 & 100 & 100 \\
\hline
\end{tabular}

Source : enquête Éric Gobe 2008-2009.

L'exercice de hautes fonctions au sein de la khaliyya permettait de transformer les ressources politiques en clientèle. II faisait du membre du parti-État un notable que le justiciable voyait comme une personnalité disposant de relations susceptibles de faire aboutir positivement son affaire. Un des avocats les plus en vue de la «cellule » évoque le lien étroit qui le lie avec sa région d'origine. Ses clients se déplaçaient pour lui rendre visite à Tunis et s'attacher ses services :

C'est mon terroir. Tout le monde me connaît, j'ai été député de K. pendant trois mandats. On vient me voir de chez moi. Les gens ont confiance en moi, ils me connaissent personnellement. On vient me voir surtout pour des affaires criminelles 25 .

Un autre membre du RCD, ancien haut responsable de la cellule, explique comment la composition de sa clientèle a évolué :

Au début de ma carrière, mes clients c'était surtout des personnes du privé. Je n'avais pas beaucoup travaillé le pénal. Mon patron de stage faisait beaucoup de civil et de contrats, des contrats de la conservation foncière. C'était des individus. Ça s'est renversé maintenant, je n'ai plus de privés, je travaille beaucoup avec des sociétés publiques, des assurances. J'ai fait une année de gouverneur, juste après j'ai fait deux mandats de l'Assemblée nationale, c'est-à-dire 10 ans de député. J'ai connu beaucoup de monde dans toute la société tunisienne. Chez les pauvres comme chez les moins pauvres. Ensuite, je me suis introduit dans le sport, j'ai fait trois fois président de la Fédération de ... Tout cela a créé un environnement favorable autour de ma personne 26 .

Par ailleurs, les « grands » praticiens du droit des affaires ayant créé des sociétés d'avocats avaient, parmi leurs associés, un membre du RCD. Mais ce n'était pas dans l'optique de se voir allouer une clientèle d'entreprises publiques par le partiÉtat. L'associé, membre du RCD, constituait une assurance contre une éventuelle intrusion du pouvoir politique dans les affaires du cabinet et jouait le rôle d'un intermédiaire facilitant le contact avec une clientèle internationale 27 . Autrement dit, pour

25. Entretien, octobre 2009. Cet avocat réalisait en 2009 plus de la moitié de son chiffre d'affaires avec des entreprises publiques.

26. Id.

27. Ces avocats sont amenés à traiter des dossiers se rapportant aux investissements internationaux, aux privatisations, aux appels d'offre internationaux ou encore à l'arbitrage, etc. 
accumuler leurs ressources économiques, les cabinets d'avocats d'affaires devaient au minimum afficher leur neutralité vis-vis du pouvoir politique. Les pressions amicales exercées sur certains clients et les éventuelles interventions des « services fiscaux » 28 incitaient ces avocats (pas seulement eux d'ailleurs) à chercher un « accommodement négocié » avec les gouvernants 29 .

Les cadres de la cellule étaient régulièrement récompensés pour les services rendus aux gouvernants. Ils se retrouvaient élus ou nommés au Parlement à un moment ou à un autre de leur carrière. En 2006, le président de la République avait nommé plusieurs cadres de la khaliyya au sein de la toute nouvelle Chambre des conseillers, innovation institutionnelle créant une seconde chambre au Parlement, destinée à élargir les bases du clientélisme d'État tunisien 30 . Certains membres de la khaliyya ont été nommés gouverneurs, tandis que d'autres ont été élus sur les listes du RCD à la Chambre des députés lors des dernières élections législatives de 2009.

Par conséquent, appartenir à l'encadrement de la khaliyya permettait de connaître, en moins d'une dizaine d'années généralement, une ascension sociale et une réussite professionnelle débouchant sur un accroissement de ses ressources financières. Toutefois, pour se voir ainsi récompensé, il convenait d'exécuter les directives du président la République et de participer ainsi aux mécanismes de discipline et de surveillance mis en place par les gouvernants.

Comptant, semble-t-il, environ 500 membres en 2009 31, la cellule opérait aussi bien à l'étranger que sur le territoire national. À l'étranger, les membres de la khaliyya étaient chargés de défendre l'image du régime, notamment lors des conférences et symposiums organisés par les associations arabes et internationales d'avocats. II s'agissait de surveiller les activités des représentants élus de l'Ordre présents à ces conférences et symposiums, notamment ceux affichant une étiquette d'opposants, pour éventuellement les contredire lorsque le besoin s'en faisait sentir 32. Sur le territoire tunisien, les membres de la cellule déployaient leur action afin de s'opposer aux velléités d'indépendance de l'Ordre en tentant de faire élire au poste de bâtonnier et au Conseil de l'ordre les candidats du RCD ou, tout au moins, des personnalités proches du parti présidentiel et, faute de mieux, des avocats à même d'afficher une « neutralité » à l'égard du pouvoir.

Last but not least, les avocats du parti présidentiel étaient utilisés par le pouvoir aux fins d'instrumentalisation de l'institution judiciaire. Ils étaient chargés de saisir la justice et d'utiliser l'arme de la procédure pour limiter autant que faire se peut toute

28. Béatrice HIBOU, “Nous ne prendrons jamais le maquis". Entrepreneurs et politique ", Politix, 21 (84), 2008, p. 115-141 (voir p. 118).

29. Béatrice HiBOU, La force de l'obéissance. Économie politique de la répression en Tunisie, op. cit.

30. Cf. Abdelwahab HANI, «Qui trouve-t-on dans la liste clientéliste du président ? »,

http://nawaat.org/portail/2005/08/01/que-trouve-t-on-dans-la-liste-clienteliste-du-president/, consulté le 2 mai 2006

31. Nous n'avons pas eu accès à des statistiques officielles. C'est le chiffre que donnaient souvent tant les opposants que les membres du RCD rencontrés.

32. Abderraouf AYADI, "La cellule des avocats: mission sécuritaire », http://ayadi.messagemonster.com, consulté le 6 janvier 2005 (en arabe). 
volonté d'émancipation trop forte. L'objectif était de faire condamner les dirigeants de l'Ordre ayant des postures contestataires. Cette technique de domestication des institutions de la "société civile » avait l'avantage de participer à la fiction de l'existence d'un État de droit puisque c'était des membres de l'organisation qui saisissaient la justice et que les procédures engagées en justice se rapportaient à des affaires internes concernant lesdits membres.

Si les avocats du parti-État avaient un quasi-monopole sur la représentation des sociétés publiques, ceux qui affichaient leur opposition au pouvoir du président Ben Ali avaient une clientèle composée uniquement d'individus. Leur engagement politique n'avait pas du tout le même caractère que celui des avocats du RCD. Bien au contraire, il avait un coût financier et prolongeait dans leur engagement professionnel leur militantisme politique oppositionnel dans l'extrême gauche ou l'islam politique 33 . Cette situation les cantonnait à certains domaines du droit (principalement le statut personnel, la rédaction de contrats immobiliers, le droit du travail et le droit pénal) et les excluait du champ du droit des affaires. Ces avocats opposants étaient généralement conduits à s'engager dans des procès pénaux à connotation politique :

Je suis comme un médecin généraliste. Les trois quarts de ma clientèle, ce sont mes amis et des parents qui ont des affaires. Ensuite, je défends les syndicalistes renvoyés, c'est-à-dire que je fais un peu de droit du travail et que je plaide devant les prud'hommes. D'un point de vue matériel, je suis resté un professeur d'enseignement secondaire. Heureusement que ma femme travaille. Mais tout ça m'a fait gagner en indépendance. Ce qui m'a fait remarquer, ce sont les plaidoiries politiques qui sont toujours poussées face au juge. Je le mets devant ses responsabilités. La procédure est nulle, le fonds est politique, la torture est présente. Mais dans les procès politiques, je ne demande pas d'honoraires 34 .

Pour autant, peut-on considérer que l'activité de ces avocats engagés dans l'opposition au régime de Ben Ali relevait de la problématique du cause lawyering ? Cette notion, qui s'est particulièrement développée à partir des années 1970 à mesure que s'affirmaient des droits subjectifs tels que le droit des femmes, le droit des consommateurs, ou encore le droit de l'environnement, etc. ${ }^{35}$, est utilisée par les auteurs « comme une catégorie et comme un outil analytique permettant à la fois de délimiter une population de juristes et un type d'activités spécifiques, orientées vers une cause politiquement ou socialement marquée » 36 .

Les avocats tunisiens opposants sous Ben Ali se situent, à cet égard, dans un entre-deux, relevant à la fois des « avocats politiques » décrits par Lucien Karpik et

33. Nous avons rencontré 13 avocats membres de l'opposition : 4 d'obédience islamiste et 9 de gauche ou d'extrême gauche.

34. Les avocats opposants se situaient dans la tranche la plus basse des chiffres d'affaires (de 10000 à 30000 DT).

35. Austin Sarat et Stuart A Scheingold (eds.), Cause Lawyering : Political Commitments and Professional Responsibilities, New York: Oxford University Press, 1998 ; ID. (eds.), The Worlds Cause Lawyers Make: Structure and Agency in Legal Practice, Stanford (Ca.) : Stanford Law and Politics, 2005 ; ID. (eds.), Cause Lawyering and the State in a Global Era, New York : Oxford University Press, 2001.

36. Liora ISRAËL, "Présentation du dossier "La justice comme espace politique. Trois études de cas : Israël, Inde, Argentine" », Droit et Société, 55, 2003, p. 600. En français, on pourra consulter également, de ce même auteur : ID., Usages militants du droit dans l'arène judiciaire : le cause lawyering ", Droit et Société, 49, 2001, p. 793-824 ; ID., L'arme du droit, Paris, Presses de Sciences Po, 2009. 
Terence Haliday 37 et des cause lawyers. Certes, leur activité politique militante est alors indissociable de leur activité professionnelle, car ils plaident systématiquement lors des procès politiques. Utilisant leurs compétences professionnelles au service d'une « cause autre que - ou plus grande que - celle des intérêts de leurs clients afin de remettre en cause certains aspects du statu quo $\gg 38$, ils se distinguent de leurs confrères « traditionnels » qui ajustent leurs « pratiques de manière à s'adapter aux besoins du client dans le cadre des rapports de pouvoir existants " 39 . Mais leurs plaidoiries devant les tribunaux ont une visée plus générale que la défense d'une cause : leur objectif est alors de défendre les valeurs libérales revendiquées par la profession en se centrant sur la défense des «droits individuels » et des «libertés fondamentales » 40 (dénonciation de la torture, du non-respect des procédures, des lois liberticides comme celle sur le terrorisme, etc.) contre l'arbitraire de l'État autoritaire. Si, de manière générale, ils se constituent plus facilement pour des justiciables appartenant à des catégories dominées, ils ne sont pas pour autant spécialisés dans la défense d'une cause particulière.

Les contraintes imposées par le pouvoir ont pu conduire les avocats politiques à une forme de spécialisation dans le domaine pénal. Un ancien cadre du parti islamiste Ennahda est devenu pénaliste après sa sortie de prison dans les années 1990. En effet, le contexte de coercition du début de la décennie 1990 avait suscité la peur de sa « clientèle civile ». Les individus qui venaient, avant son arrestation, lui confier des affaires civiles, ainsi que toutes les sociétés de son portefeuille, avaient déserté le cabinet.

Avant d'entrer en prison, j'avais des sociétés avec lesquelles je travaillais. Je faisais surtout du civil, assez peu de pénal. Je n'étais pas pénaliste. J'avais une grosse clientèle avec des sociétés et des personnes physiques. Lorsque je suis sorti de prison, plus personne n'osait me donner ses affaires civiles. Je suis islamiste, j'étais attaqué par le pouvoir, ils avaient peur pour leurs intérêts. Qui est venu me voir? Les gens qui connaissaient mon nom, des gens qui me respectaient pour mes convictions religieuses et politiques ou des gens qui n'avaient rien à perdre, c'est-à-dire les gens qui ont des affaires pénales, qui n'ont pas d'intérêts à perdre. II y avait des gens qui étaient ignorants du danger encouru. Ce sont des Tunisiens de la basse classe, du peuple, ce sont ceux qui sont plus impliqués que d'autres dans les affaires pénales, les pauvres. Mais ce sont des clients passagers, parce que celui qui passe dans votre étude pour une affaire de vol, de vol qualifié ou de meurtre, ce n'est pas un client permanent. II ne passe qu'une fois, deux fois ou trois fois en cas de récidive. Ce sont toujours des nouveaux qui passent me voir. J'ai quelques clients qui reviennent parce qu'ils ont des affaires de

37. Terence C. Halliday, Lucien KARPIK et Malcom M. FeELEY, « Legal Complex and Struggles for Political Liberalism ", in ID., Fighting for Political Freedom. Comparative Studies of the Legal Complex and Political Liberalism, Oxford, Portland (Ore.) : Hart, coll. « Onãti International Series in Law and Society », 2007, p. 140 .

38. Lisa HAJJAR, «From the Fight for Legal Rights to the Promotion of Human Rights. Israeli and Palestinian Cause Lawyers in the Trenches of Globalization ", in Austin SARAT et Stuart A. ScheINGold (eds.), Cause Lawyering and the State in a Global Era, Oxford, New York : Oxford University Press, 2001, p. 68.

39. Ibid.

40. Lucien KARPIK, « Les professions libérales sont-elles solubles dans le marché ? », in Thomas LE BIANIC et Antoine VION (dir.), Action publique et légitimités professionnelles, Paris : LGDJ, 2008, p. 284. 
divorce ou de pension alimentaire, des affaires sociales ; ils reviennent me voir, c'est le paquet de clients qui reste attaché à l'étude 41 .

Outre les pressions sur la clientèle, les autorités utilisaient régulièrement l'arme fiscale contre les avocats militants de l'opposition : l'ensemble des défenseurs militants rencontrés avaient vu, entre 2005 et 2008, les inspecteurs des impôts leur rendre visite et opérer un redressement fiscal.

\section{III. « Petite samsara » et contrôle social de la profession}

Si le contrôle fiscal faisait partie de la panoplie des outils utilisés par le régime de Ben Ali pour « surveiller et punir » les avocats récalcitrants, le pouvoir disposait aussi de moyens indirects, mais non moins efficaces, de contrôle de la profession. II laissait se développer une pratique illégale de la profession chez les avocats généralistes traitant de «petites affaires » banalisées, notamment au pénal. Ils recouraient au service de samsars, autrement dit d'agents mandatés officieusement qui, en violation de la loi, font du racolage et alimentent en clientèle certains avocats. Cette forme de courtage illégal, dénommé par nos enquêtés "petite samsara " (al-samsara alsoughra) n'est pas de même nature que la " grande samsara " exercée par le partiÉtat. Mais l'État autoritaire, en tolérant le développement de cette pratique professionnelle, se donnait les moyens de sanctionner légalement les avocats qui auraient eu des velléités de protestation.

Nous n'avons pas de données quantitatives sur la pratique de la petite samsara (et pour cause !), mais elle était perçue comme un phénomène gangrénant la profession. Elle préoccupait au plus haut point les avocats tunisiens qui y voyaient une forme de concurrence déloyale : près de $90 \%$ des avocats interrogés dans le cadre de l'enquête quantitative pensaient que la samsara était un problème pour la profession.

Par delà le cas spécifique de la Tunisie sous la présidence Ben Ali, les courtiers constituent une ressource sociale pour les avocats qui en sont initialement peu dotés. Ces derniers pallient ainsi la faiblesse de leurs ressources sociales initiales 42 en développant un type d'échange clandestin avec diverses catégories d'individus gravitant autour des tribunaux. Cette pratique est le propre des barreaux où le marché est particulièrement concurrentiel et volatil. La distinction opérée par Lucien Karpik entre une concurrence par la qualité et une concurrence par les prix pour analyser le fonctionnement du marché des avocats permet de comprendre le développement de la

41. Entretien avec un avocat pénaliste, ancien cadre d'Ennahda, octobre 2008.

42. Au sens où l'entend Nan LIN, «Les ressources sociales : une théorie du capital social », Revue française de sociologie, 36 (4), 1995, p. 685-704 (voir p. 687). II définit les ressources comme « des biens dont la valeur est déterminée socialement et dont la possession permet à l'individu de survivre ou de préserver des acquis ". Ce faisant, Nan Lin développe une théorie qui se concentre sur les ressources contenues dans le réseau social et "sur la manière dont l'action individuelle bénéficie de l'accès et de l'utilisation de ces ressources ». Par conséquent, "le capital social est l'investissement d'un individu dans ses relations avec les autres » (p. 701). Dans la mesure où Nan Lin considère que les ressources sociales constituent l'« élément central du capital social », nous utiliserons l'un pour l'autre. Par ailleurs, nous considérons que les ressources politiques constituent une espèce particulière de capital social dans la mesure où ce sont des moyens mobilisables, plus précisément des relations dans la sphère politique, susceptibles d'augmenter les chances pour l'individu d'atteindre un objectif, en l'occurrence de constituer une clientèle. 
samsara, elle-même révélatrice de la marchandisation d'un espace intermédiaire entre l'avocat et le client. Dans le premier cas de figure, l'offre des services juridiques est diversifiée en fonction des qualités, et par conséquent le choix d'un avocat par le client s'opère par « un jugement dont la validité dépend des mécanismes qui, comme le réseau et la confiance, permettent de réduire l'incertitude de la qualité » 43 . Dans le second cas, « le prix représente le seul trait différentiel, et ses variations assurent l'ajustement réciproque de l'offre et de la demande »44. Dans la Tunisie de Ben Ali, cette forme de concurrence touchait, au premier chef, le segment dominé de la profession, c'est-à-dire le «bas barreau » tunisien où le nombre de jeunes avocats confrontés à la recherche d'une clientèle individuelle avait explosé depuis le début des années 1990. Elle était particulièrement présente en Tunisie, comme sous d'autres cieux, dans des domaines du droit peu prestigieux (le petit pénal) et concernait des dossiers simples à traiter relevant pour la plupart des accidents de la route 45 .

L'accroissement de la concurrence exerçait une pression à la baisse des honoraires dans les affaires les plus simples. Sept avocats généralistes sur dix se plaignaient des clients nomades qui faisaient le tour des cabinets pour se renseigner sur les tarifs pratiqués :

Généralement, un client vient dans ton cabinet, il a une affaire pas très compliquée. J'ai eu récemment un client qui est venu pour une centaine de chèques impayés. II s'était renseigné avant sur les tarifs et m'a fait comprendre qu'il irait voir quelqu'un d'autre si besoin parce qu'il savait que c'était une affaire assez facile et qu'il allait pouvoir faire travailler l'affaire à $60 \mathrm{DT}$ au lieu de $150 \mathrm{DT}$. Là, on négocie. On peut descendre jusqu'à $100 \mathrm{DT}$; en dessous, c'est vraiment une misère 46

Mais le recours par les avocats à ce type particulier de courtiers n'est pas le propre de la Tunisie de Ben Ali. Jerome E. Carlin 47 , dans son étude classique sur les avocats de Chicago exerçant à titre individuel, décrit les relations de courtage entre les avocats et divers intermédiaires dans le domaine des accidents de la route: certains avocats ont développé des réseaux de contacts au sein des forces de police et du corps médical travaillant dans les hôpitaux pour approcher le plus rapidement possible les victimes d'accidents de la circulation et leur faire signer un contrat de représentation avec un avocat donné. De son côté, J. S. Gandhi $48 \mathrm{a}$, dans sa thèse, analysé les liens entre la réussite professionnelle de certaines catégories d'avocats indiens et le recours à des intermédiaires chargés de « recruter » des clients.

43. Lucien KARPIK, Les avocats. Entre l'État, le public et le marché (XIII $-X X^{e}$ siècle), Paris : Gallimard, 1995, p. 273.

44. Lucien Karpik (ibid., p. 275) précise que la concurrence par les prix s'impose lorsque le service proposé est standardisé.

45. Ibid., p. 275

46. Entretien avec un avocat généraliste au chiffre d'affaires de 30000 DT, octobre 2008 .

47. Jerome E. CARLIN, Lawyers on their Own. A Study of Individual Practitioners in Chicago, New Brunswick (N.J.) : Rutgers University Press, 1962, p. 87

48. Jogindra Singh GANDHI, Lawyers and Touts. A Study in the Sociology of Legal Profession, Dehli : Hindustan Pub. Corp., 1982. 
Dans le contexte du barreau tunisien, le samsar fait précisément référence à une catégorie de personnes utilisées par les avocats pour obtenir des clients en échange d'une partie des honoraires qu'ils perçoivent du client. Le samsar opère une médiation entre deux parties, l'une souhaitant vendre un service juridique et l'autre désirant l'acheter. Mais à la différence d'un simple courtier, le samsar fait du racolage ou du rabattage (istijlab). II touche une commission versée par l'avocat, le fournisseur du service professionnel, mais n'est aucunement rétribué par le client. Ce dernier ne le voit pas toujours comme un rabatteur. Le samsar s'efforce d'ailleurs de créer une relation de confiance avec le client potentiel et se présente comme une personne susceptible de rendre un service à titre amical, guidée par le désir de donner un «bon conseil » au justiciable 49 .

Pour être plus précis, on peut distinguer deux formes de samsara: le processus de racolage se produit soit dans une interaction directe indépendamment d'un rapport institutionnel, soit à travers une transaction qui prend place dans un cadre institutionnel plus ou moins contraignant pour le client 50 .

La première forme se déroule la plupart du temps au Palais de justice. Un justiciable, souvent de condition modeste, se rend au tribunal pour se présenter à une audience où le ministère de l'avocat n'est pas obligatoire, pour récupérer la copie d'un jugement ou encore trouver une personne (un écrivain public, par exemple) susceptible de lui rédiger une requête. II est alors accosté dans l'enceinte ou au voisinage du tribunal par un greffier, un clerc d'avocat ou encore un policier qui se propose de l'aider dans ses démarches et, par conséquent, de lui trouver l'avocat qui convient. Cela peut être aussi les parents des détenus qui vont au Palais de justice pour se renseigner sur la situation d'un proche et qui sont racolés par un samsar. Ce dernier met en contact le client et l'avocat qui a souvent un cabinet proche du tribunal mais qui peut très bien être une espèce d'avocat « ambulant » gravitant dans les cafés entourant le Palais de justice 51.

Un avocat généraliste rencontré décrivait de la manière suivante le processus de prise de contact entre le samsar et l'avocat, puis entre le samsar et le client :

Qu'est-ce qui se passe ? Les avocats sont de plus en plus nombreux, ils sont de plus en plus jeunes, ils crèvent la faim. II y a des flics dans les tribunaux chargés de les repérer. Ils les repèrent et leur disent : "Tu veux travailler, tu veux nourrir ta famille, et bien on va t'envoyer des clients. "Les clients, eux, sont dépassés par les événements devant le Palais de justice. Un de leurs proches a été arrêté ou est au commissariat de police. La famille ou les proches sont orientés vers Maître un tel, jeune avocat fraîchement débarqué dans la profession. Maître un tel demande 400 dinars pour l'affaire. II en

49. Les avocats généralistes et les deux spécialistes du pénal de l'échantillon qualitatif m'ont décrit les mécanismes de la samsara de la même façon. Je leur ai demandé si eux-mêmes l'avaient pratiquée à un moment de leur carrière. Tous m'ont répondu négativement, ce qui n'est guère surprenant dans la mesure où cette pratique est illégale et fortement stigmatisée tant par les avocats que par les instances ordinales.

50. Jogindra Singh GANDHI, Lawyers and Touts. A Study in the Sociology of Legal Profession, op. cit., p. 113.

51. $6 \%$ des avocats n'ont pas d'employé (source : enquête Éric Gobe, 2008-2009). Certains d'entre eux n'ont pas de cabinet, tout au moins ils le domicilient à leur adresse personnelle et n'y reçoivent pas de clients. Ils travaillent à partir des cafés environnant les tribunaux, soit en utilisant des samsars pour attirer des clients, soit en faisant du racolage directement. 
donne 100 au flic, 100 au rabatteur, 100 au greffier. Et, du jour au lendemain, Maître un tel se met à plaider au moins six ou sept dossiers de correctionnel par jour 52 .

Une seconde forme de samsara s'inscrit dans un cadre institutionnel qui précède l'interaction et la transaction entre le samsar et le justiciable, puis entre l'avocat et le justiciable. Dans cette forme de samsara, le rabatteur occupe une fonction au sein de l'institution (la prison, l'hôpital, le commissariat de police) et peut exercer sur le client une forte pression morale, voire physique.

La samsara qui est évoquée de façon systématique par les avocats interrogés est celle dans laquelle les fonctionnaires de police et les agents de la garde nationale (l'équivalent de la gendarmerie) sont impliqués au profit de certains avocats pratiquant le droit pénal. Des justiciables en état d'arrestation dans un commissariat sont dirigés vers un avocat donné. La commission est collectée auprès de l'avocat par un policier qui la partage avec ses collègues. Ici le samsar est en position de faire l'intermédiaire en raison de la vulnérabilité du client : il occupe une position d'autorité au sein d'une institution. Un individu placé en état d'arrestation est à la merci du fonctionnaire de police qui peut le harceler de différentes manières. On a affaire à un mode de persuasion bien plus coercitif qu'amical qui n'est possible qu'en raison des positions fortement asymétriques occupées respectivement par le samsar et le détenu.

On retrouve une asymétrie équivalente dans les prisons où les agents pénitentiaires peuvent faire valoir à la personne placée en état d'arrestation qu'ils connaissent tel ou tel excellent avocat, lequel de surcroît connaît bien les juges et est susceptible d'obtenir un jugement favorable.

Le domaine des accidents de la route est propice au développement de la samsara. En général, elle implique la participation de quatre ou cinq acteurs : l'avocat bien évidemment, l'agent de police ou de la garde nationale, l'ambulancier, le médecin ou l'infirmier. Ces derniers acteurs sollicitent les personnes hospitalisées en faisant valoir que le PV sera rédigé en leur faveur par le fonctionnaire de police ou que les expertises iront dans un sens favorable.

Dans les années 1990-2000, le conseil de discipline de l'Ordre des avocats a parfois sanctionné sévèrement les avocats impliqués, mais la cour d'appel a systématiquement minoré ou cassé les mesures disciplinaires 53 . II s'agissait, en premier lieu, pour le régime de Ben Ali de ne pas déstabiliser le ministère de l'Intérieur, cœur de l'appareil répressif : une partie de ses agents (les policiers) étaient les principaux bénéficiaires des ressources financières générées par la samsara. Mais l'existence de la samsara présentait aussi certains avantages pour les gouvernants au regard du contrôle et de la clientélisation d'une partie de la profession. Certains membres du RCD qui n'avaient pas accès aux institutions publiques pouvaient, sans être inquiétés, avoir recours à des samsars. En échange, ils devaient rendre des services aux gouvernants, notamment en portant plainte contre leurs confrères membres de l'opposition, les accusant d'avoir exercé à leur encontre une violence physique. Dans

52. Entretien avec un avocat généraliste, octobre 2009.

53. Entretiens avec deux anciens présidents des sections de Sousse et de Sfax, juillet 2008 et octobre 2009. 
de telles conditions, il leur était difficile d'exprimer un désir d'autonomie et d'avoir des velléités d'indépendance.

Le développement de la samsara était perçu par la majorité des avocats comme une conséquence de la pression concurrentielle croissante au sein de la profession, due en partie au rétrécissement du « champ d'intervention de l'avocat », c'est-à-dire à l'amputation de leur « territoire professionnel » 54 .

Par conséquent, une des revendications récurrentes des instances représentatives du barreau sous la présidence de Ben Ali a été d'accroître le territoire professionnel des avocats au détriment des professions concurrentes. Ces exigences n'ont guère rencontré d'écho favorable chez les gouvernants. Mais, dans le même temps, la redéfinition des territoires professionnels a constitué, dans les années 1990-2000, un enjeu de négociation entre les porte-parole de la profession et les gouvernants.

\section{Remodeler le territoire professionnel des avocats}

Jusqu'à la chute du régime de Ben Ali, les instances ordinales ont revendiqué, sans relâche, la mise en œuvre d'une politique qui permette aux avocats d'augmenter leur potentiel d'acquisition de ressources matérielles en élargissant leur territoire professionnel. En effet, le ministère de l'avocat n'était pas obligatoire dans un certain nombre de matières juridiques, plus particulièrement dans les petites affaires. C'était le cas en matière correctionnelle, dans les affaires de justice cantonale dont le montant était inférieur à 7000 DT, et celles dans le domaine fiscal pour des montants n'excédant pas 25000 DT.

Élargir le «champ d'intervention de l'avocat », autrement dit annexer de nouveaux «territoires professionnels », était devenu la principale revendication formulée par les avocats (tableau 3). Mais c'était surtout une demande des avocats situés au bas de la hiérarchie du barreau (entre 10000 et 30000 DT). Pour des professionnels soumis à un régime de concurrence par les prix, confrontés à une clientèle de condition plutôt modeste, la principale mesure à prendre pour améliorer la situation de la profession était d'élargir le champ. Cette mesure était vue comme un moyen de faire baisser la pression concurrentielle sur un segment du marché où le montant de I'honoraire était un facteur clé du recours ou non à un avocat.

Dans les domaines où le ministère de l'avocat n'était pas obligatoire, le justiciable pouvait préparer son dossier seul ou avoir recours à des individus appartenant à d'autres corps du personnel judiciaire (notaires, huissiers, greffiers) ou à des professions pouvant se prévaloir de compétences juridiques (experts-comptables, conseillers juridiques, conseillers fiscaux, écrivains publics). Les avocats généralistes percevaient de façon particulièrement aigüe la concurrence des professions voisines accusées de «braconner»sur leur territoire professionnel 55. Ils revendiquaient, pour reprendre la formulation d'Andrew Abbot, un territoire professionnel exclusif (claim of jurisdiction) dont il s'agit de s'assurer le contrôle par un discours ayant pour

54. Andrew Аввотт, The System of Profession. An Essay on the Division of Expert Labor, Chicago, Londres : University of Chicago Press, 1988.

55. Ibid., p. 248 
objectif d'établir le caractère naturel et nécessaire de leur expertise sur cet espace social, alors qu'il n'est que « contingent, construit et précaire » 56 .

Les avocats considéraient qu'il convenait de préserver le «périmètre du droit »en l'occurrence celui des avocats 57 - des «intrus» et des «parasites» 58 . Pour ce faire, les avocats souhaitaient disposer de l'exclusivité de la rédaction de certains contrats comme, par exemple, celle du statut des sociétés ou des contrats de vente immobilière. Par ailleurs, rendre obligatoire le ministère de l'avocat devant la justice cantonale, en matière correctionnelle ou prud'homale, contribuerait à évincer du marché des services juridiques les écrivains publics, les notaires, les huissiers ou encore les greffiers des tribunaux, c'est-à-dire les principaux concurrents des avocats généralistes. II s'agissait également d'éliminer de l'activité de conseil les conseillers juridiques qui, en Tunisie, concurrencent également les avocats du «bas barreau ». En effet, ces derniers, titulaires d'une maîtrise en droit, ont échoué au CAPA ou n'ont pas pu intégrer un troisième cycle de droit. Aussi se sont-ils installés comme conseillers juridiques, car aucun diplôme n'est exigé par la loi pour ouvrir un cabinet, même si de facto l'administration exige que l'individu souhaitant s'installer soit titulaire d'une maîtrise en droit. Ce faisant, le régime de Ben Ali promouvait le développement d'une forme d'auto-emploi d'une catégorie de diplômés fortement touchée par le chômage. Contrairement à ce qui a pu exister en France pendant quasiment tout le $x x^{e}$ siècle, il n'y a jamais eu en Tunisie d'élites du conseil juridique pouvant se prévaloir de titres scolaires équivalents à ceux des avocats 59 .

Par conséquent, les spécialistes et les généralistes n'avaient pas la même perception des professions concurrentes. Les premiers, dominés par les avocats d'affaires, percevaient l'expert-comptable, l'homme du chiffre, comme venant empiéter sur leur territoire professionnel, tandis que les seconds désignaient comme leurs principaux concurrents les professionnels intervenant dans les petites affaires civiles où le ministère de l'avocat n'est pas obligatoire (les huissiers, les notaires et les écrivains publics).

56. Ibid. ; Yves DeZALAY, Marchands de droit. La restructuration de l'ordre juridique international par les multinationales du droit, Paris : Fayard, 1992, p. 174.

57. Laurent WILLEMEZ, «Le "périmètre" des savoirs des avocats : lutte de concurrence et représentation professionnelle dans les conseils de prud'hommes ", $4^{\mathrm{e}}$ Conférence intermédiaire du Comité de recherche "Sociologie des groupes professionnels " de l'Association internationale de sociologie, Université de Versailles Saint-Quentin, 22 septembre 2004.

58. Mémorandum du Conseil de l'ordre national des avocats sur la réforme de la situation de la profession (en arabe), 2004.

59. Anne BoIGeOL et Yves DeZALAY, « De l'agent d'affaires au barreau : les conseils juridiques et la construction d'un espace professionnel », Genèses, 27, 1997, p. 49-68 (voir p. 54). 
Tableau 3

Les professions perçues comme concurrentes selon que l'avocat est généraliste ou spécialiste (Plusieurs réponses sont possibles, les pourcentages ne s'additionnent pas; $n=531$ )

\begin{tabular}{|l|c|c|c|}
\hline & Généraliste & Spécialiste & Total \\
\hline $\begin{array}{l}\text { Notaire, huissier, } \\
\text { écrivain public }\end{array}$ & 44 & 23 & 40 \\
\hline $\begin{array}{l}\text { Expert-comptable, } \\
\text { comptable }\end{array}$ & 29 & 26 & 33 \\
\hline $\begin{array}{l}\text { Conseiller juridique, } \\
\text { conseiller fiscal }\end{array}$ & 37 & 4 & 7 \\
\hline $\begin{array}{l}\text { Samsar, greffier, } \\
\text { clerc d'avocat }\end{array}$ & 8 & 3 & 6 \\
\hline Administration & 6 & 3 & 4 \\
\hline $\begin{array}{l}\text { Société de recouvrement, } \\
\text { agent immobilier }\end{array}$ & 4 & 0 & 2 \\
\hline Autre & 3 & & \\
\hline
\end{tabular}

Source : enquête Éric Gobe 2008-2009.

Aussi les praticiens généralistes considéraient-ils que la principale mesure permettant d'améliorer la situation de la profession était l'élargissement du champ d'intervention de l'avocat, tandis que les spécialistes citaient en premier l'amélioration du fonctionnement de l'État de droit et de la formation (tableau 4) 60.

Tableau 4

Mesures prioritaires pour améliorer la situation de la profession selon que l'avocat est généraliste ou spécialiste

Plusieurs réponses sont possibles, les pourcentages ne s'additionnent pas $(n=620)$

\begin{tabular}{|l|c|c|c|}
\hline & Généraliste & Spécialiste & Total \\
\hline $\begin{array}{l}\text { Élargir le champ } \\
\text { d'intervention de l'avocat }\end{array}$ & 67 & 38 & 62 \\
\hline $\begin{array}{l}\text { Améliorer le fonctionnement } \\
\text { de l'État de droit }\end{array}$ & 31 & 41 & 33 \\
\hline Améliorer la formation & 23 & 36 & 26 \\
\hline Autre & 8 & 4 & 7 \\
\hline $\begin{array}{l}\text { Augmenter l'aide } \\
\text { juridictionnelle }\end{array}$ & 3 & 0 & 2 \\
\hline
\end{tabular}

Source : enquête Éric Gobe 2008-2009.

L'élite de la profession, c'est-à-dire les avocats spécialistes et plus particulièrement ceux spécialisés en droit des affaires, n'était bien évidemment pas concernée par les matières traitées au tribunal cantonal et, de manière générale, fréquentait assez peu les salles d'audience des tribunaux. Plus on s'élevait dans la hiérarchie

60. J'ai formulé la question de manière à ne pas avoir de problèmes avec les autorités tunisiennes. On ne peut pas parler, dans le cas de la Tunisie de Ben Ali, de l'existence d'un État de droit. 
des chiffres d'affaires, moins on considérait que l'élargissement du champ d'intervention de l'avocat était une mesure pertinente (pour les chiffres d'affaires compris entre 30000 à 70000 DT, un peu plus de la moitié ; cinq chez les spécialistes, dont les deux membres du RCD).

Aussi n'était-il guère surprenant d'entendre les avocats au sommet de la hiérarchie des chiffres d'affaires (14 sur 19) tenir un discours libéral critique vis-à-vis de la revendication de l'élargissement du champ d'intervention, considérant que c'est à l'avocat de s'imposer par ses compétences et de se rendre indispensable aux justiciables.

Face à cette revendication d'“élargissement du champ d'intervention » de l'avocat, les gouvernants ont eu un positionnement à géométrie variable en fonction de la tension qui pouvait exister entre les instances dirigeantes du barreau et les autorités et, par conséquent, selon la posture plus ou moins oppositionnelle affichée par les instances ordinales à l'égard du régime de Ben Ali.

De manière générale, le pouvoir hésitait à accéder aux revendications des avocats, celles-ci étant susceptibles de contribuer à l'élargissement de l'espace d'autonomie d'une profession judiciaire qui, dans l'exercice de sa fonction de défense, était à même de contester les logiques de l'autoritarisme (dénonciation de l'arbitraire, non-respect des procédures, violations des droits de la défense, etc.). Mais, dans le même temps, refuser de répondre positivement aux revendications des avocats alimentait la contestation au sein de la profession et, d'une certaine façon, politisait les enjeux professionnels 61 . Ce faisant, le pouvoir s'est trouvé confronté à un dilemme qui a contribué, à partir des années 2000, à placer à la tête du barreau des bâtonniers plus ou moins opposants au régime de Ben Ali.

Ainsi le vote de lois étendant ou restreignant le territoire professionnel de l'avocat a-t-il constitué pour les gouvernants un instrument à la fois de punition et de négociation pour obtenir l'allégeance du Conseil de l'ordre. Par exemple, peu après l'élection du bâtonnier Abdessatar Ben Moussa en juillet 2004, le président de la République a fait suspendre l'examen par la Chambre des députés du projet de loi relatif à l'amendement du Code des assurances considéré par les structures de l'Ordre comme un texte de loi supplémentaire restreignant le domaine d'intervention de l'avocat. En effet, ce texte « déjudiciarisait » une partie du contentieux des accidents de la route en prévoyant une phase de conciliation entre les victimes et les compagnies d'assurance avant un éventuel recours à la Justice. Mais la dégradation des relations entre le bâtonnier et les autorités à la suite de l'« affaire Abbou » 62 en mars 200563 a débouché in fine sur l'adoption du texte précédemment suspendu. En sens

61. Éric GOBE, «Corporatismes, syndicalisme et dépolitisation », in Élisabeth PICARD, La politique dans le monde arabe, Paris : Armand Colin, 2006, p. 171-192 (voir p. 190).

62. Du nom d'un avocat membre d'un parti non reconnu sous la présidence Ben Ali, le Congrès pour la République. Celui-ci a été condamné pour s'en être pris sur Internet à la personne du président de la République, bien que les motifs de la condamnation invoquent la diffusion de fausses nouvelles dans l'intention de troubler l'ordre public et une prétendue agression physique contre une avocate lors d'une conférence en 2002.

63. Éric GoBE, «The Tunisian Bar to the Test of Authoritarianism : Professional and Political Movements in Ben Ali's Tunisia (1990-2007) ", Journal of North African Studies, 15 (3), 2010, p. 333-347 (voir p. 340-342). 
contraire, la position particulièrement conciliatrice du nouveau bâtonnier et ancien opposant, Bechir Essid, à l'égard du pouvoir politique a abouti à l'adoption en 2008 d'une loi rendant le ministère de l'avocat obligatoire devant le tribunal immobilier 64 .

En fait, les avocats tunisiens subissaient sous Ben Ali les mêmes contraintes économiques que leurs confrères d'autres pays. Ils étaient notamment soumis à la concurrence plus ou moins sévère d'autres professions et étaient engagés dans d'intenses batailles territoriales. Mais, dans la Tunisie de Ben Ali, les rapports conflictuels entre barreau et le pouvoir autoritaire ont souvent conduit celui-ci à redéfinir les frontières professionnelles au détriment des avocats. Avec le départ du président Ben Ali, c'est un scénario tout différent qui s'écrit : le barreau paraît désormais suffisamment puissant pour obtenir d'un pouvoir politique de transition une recomposition des territoires professionnels en sa faveur.

\section{Conclusion. Les avocats dans la Tunisie post-Ben Ali : un nouveau pouvoir?}

La chute du régime de Ben Ali a propulsé les porte-parole de la profession, plus particulièrement le bâtonnier Abderrazak Kilani, sur le devant de la scène politique et a déclassé les avocats du RCD, privés des ressources matérielles et symboliques que leur procurait l'allégeance à l'ancien régime. Les avocats du parti-État désormais dissous font profil bas. Ils ont officiellement perdu le monopole du contentieux des sociétés publiques, le gouvernement de transition ayant pris une circulaire donnant aux PDG d'entreprises publiques la liberté de choisir leurs avocats 65 .

Dès la mise en place du gouvernement de transition, les instances ordinales se sont impliquées dans la sphère politique tunisienne en recomposition. Elles ont adopté une posture critique vis-à-vis du gouvernement de transition dirigé par Mohamed Ghanouchi, considéré comme trop lié à l'oligarchie de l'ancien régime. Un peu moins d'un mois après la fuite de Ben Ali, l'Ordre des avocats a adhéré au Conseil national de sauvegarde de la révolution, coalition hétérogène rassemblant 28 partis politiques, associations et organisations professionnelles. Les porte-parole de la profession se sont ainsi retrouvés aux côtés des leaders de la centrale syndicale, l'UGTT, des islamistes d'Ennahda, de divers groupuscules d'extrême gauche et des associations de défense des droits de l'homme et de lutte contre la torture pour exiger une forte épuration de l'appareil d'État et demander l'élection d'une assemblée constituante. La démission de Mohamed Ghanouchi le 27 février 2011 et l'acceptation par son successeur, Béji Caïd Sebsi, du principe de l'élection d'une assemblée constituante ont sonné comme une victoire pour le Conseil national de sauvegarde de la révolution.

64. Toutefois, certains avocats spécialisés dans le domaine foncier ont nuancé la lecture politique de cet élargissement du champ d'intervention de l'avocat en faisant valoir que c'est la nécessaire actualisation des titres fonciers en Tunisie, matière juridique éminemment complexe, qui avait poussé les autorités à rendre le recours à l'avocat obligatoire (entretien avec un avocat spécialisé en droit immobilier, octobre 2009).

65. Le texte du Premier ministère demande aux dirigeants des institutions publiques de recourir à des critères objectifs de compétence et de transparence pour s'attacher les services d'un avocat, indépendamment de toute liste nominative préalablement établie. Cf. Circulaire $n^{\circ} 4$ du Premier ministre adressée aux p résidents des entreprises et établissements publics, 9 février 2011 (en arabe). 
Omniprésent dans les médias tunisiens, le bâtonnier se trouve doté de nouvelles ressources politiques qu'il mobilise pour faire avancer les revendications professionnelles formulées par les instances ordinales sous l'ancien régime. L'objectif pour le bâtonnier et ses soutiens est de réorganiser la profession dans le sens d'un rehaussement de son statut, d'un approfondissement de son autonomie et d'un élargissement de son territoire professionnel.

L'autorisation donnée par le Parlement au président de la République par intérim de prendre des décrets-lois a incité le bâtonnier à accélérer l'élaboration d'un projet de loi organisant la profession d'avocats mis à l'étude depuis juillet 2010 au sein d'une commission. Cette démarche inspirée par Abderrazak Kilani n'a pas manqué de susciter des critiques au sein du barreau. Certains anciens et actuels responsables du Conseil de l'ordre l'ont contestée. Le bâtonnier a consulté les avocats par référendum, le 10 mars 2011, sur le projet de loi. En ayant recours à ce mode inédit de consultation des avocats, il a ignoré les instances de consultation et de prise de décision de la profession que sont le Conseil de l'ordre, les conférences de sections ainsi que les assemblées générales ordinaires ou extraordinaires. Les avocats votants n'en ont pas moins approuvé massivement le projet de loi 66 , il est vrai tout à l'avantage de la profession. L'article $1^{\text {er }}$ du projet de 2011 en fait plus que de simples auxiliaires de justice: les avocats ne constituent plus seulement « une profession libérale et indépendante ayant pour but d'aider à l'instauration de la justice » (formulation de la loi de 1989), mais un corps qui «participe à l'établissement de la justice et qui défend les libertés et les droits humains » 67 .

Mais par-delà le rehaussement du statut symbolique de la profession, certaines dispositions du texte sont apparues aux porte-parole des magistrats et des principales professions libérales concurrentes des avocats comme une atteinte intolérable à leurs prérogatives. Les premiers ont protesté contre l'article 3 du projet qui les privait du privilège d'accéder directement au barreau à leur retraite. Leur mobilisation a rencontré un certain succès puisque la version finale du décret-loi promulgué fin août 2011 prévoit que ceux qui ont exercé la magistrature pendant dix ans peuvent s'inscrire au tableau de l'Ordre.

Quant aux seconds (experts-comptables, notaires, conseillers fiscaux, etc.), ils ont critiqué l'article 2 qui attribue à l'avocat l'exclusivité de certaines missions. Le texte prévoit que « l'avocat est le seul habilité à représenter les parties, à les assister par des conseils et consultations juridiques, à accomplir les procédures requises et à les défendre devant les tribunaux et toutes les instances judiciaires, administratives et disciplinaires, ainsi que devant l'officier de police judiciaire ». Les professions concurrentes des avocats craignent que l'énoncé de cette disposition signifie qu'elles n'auront plus le droit de conseiller leurs clients en matière juridique, comptable ou fiscale et qu'elles ne pourront plus accomplir de formalités administratives pour le compte de ceux-ci.

66. Le taux de participation au référendum est d'environ $40 \%$. Il est comparable à la participation aux élections professionnelles qui oscille entre 40 et $50 \%$. Le oui l'a emporté avec près de $70 \%$ des voix.

67. JORT, Décret $n^{\circ} 79$ du 20 août 2011 se rapportant à l'orga nisation de la profession d'avocat, 23 août 2011, p. 1591 
Les $2^{\mathrm{e}}$ et $3^{\mathrm{e}}$ alinéas de l'article 2 ont été encore plus contestés par les représentants des professions concurrentes qui ont exigé l'annulation pure et simple du projet de décret-loi. En effet, le texte attribue à l'avocat l'exclusivité de « la rédaction des statuts de sociétés et de l'augmentation ou de réduction de leur capital, chaque fois qu'il s'agit d'un apport en fond de commerce ». II leur donne également l'exclusivité de la « rédaction des contrats, des actes translatifs de propriétés immobilières, des contrats de participation immobilière dans le capital d'une société commerciale à l'exception de ceux qui ont été attribués expressément aux notaires et aux rédacteurs de la Conservation foncière ».

Les professions libérales concurrentes, qui se sont opposées au projet de décretloi, n'ont pas rencontré le même succès que les magistrats. Face à leur mouvement de protestation, le bâtonnier et certains avocats ont mis en avant leur légitimité révolutionnaire. En mars 2011, dans un entretien donné au quotidien Le Temps, Abderrazak Kilani rappelait à ses contradicteurs "qu'il y a à peine deux mois, seuls les avocats avaient brisé le mur du silence et de la peur pour dénoncer les dérives de Ben Ali ». Les autres professions sont ainsi renvoyées à leur non-implication dans les mouvements de protestation contre l'ancien régime.

Le barreau semble en position de force vis-à-vis des professions concurrentes accusées d'avoir bénéficié d'un traitement de faveur à l'époque de Ben Ali. Un sentiment de revanche habite désormais certains avocats. II apparaît clairement que le bâtonnier a su tirer profit de l'effondrement du régime autoritaire pour s'impliquer directement dans l'espace politique tunisien en voie de recomposition. II a su construire un véritable geste révolutionnaire qui se prolonge jusqu'à aujourd'hui : les avocats voués à la défense des droits de l'homme et à l'instauration de la justice constituent désormais « une garantie morale pour le renforcement des droits et des libertés à un moment où les fantômes de l'ancien régime rôdent toujours et où la tentation de les domestiquer représente encore un risque réel dans notre jeune démocratie » 68 . Garantie par le décret-loi, l'immunité pénale dont bénéficient les avocats lorsqu'ils plaident devant les tribunaux est censée leur permettre d'exercer pleinement «leur rôle naturel de défenseur des droits et des libertés et de contribuer à la préservation des acquis de la révolution $» 69$.

Si l'ère Ben Ali est définitivement close, il convient de ne pas tirer des conclusions hâtives des dernières évolutions, toutes favorables au renforcement du pouvoir professionnel des avocats. En effet, la conjoncture politique demeure extrêmement fluide et nous ne connaissons pas les caractéristiques du nouveau régime politique qui est en gestation. Ainsi, dans un espace politique en recomposition, il est difficile d'anticiper les processus de construction de nouvelles légitimités professionnelles et la capacité des diverses professions à protéger ou étendre leur territoire professionnel.

68. Tribune du bâtonnier Abderrazak Kilani, Le Temps, 20 avril 2011.

69. Id. 